\title{
Influence Study of the Viscoelastic Fluids Features in Drag Reduction in Laminar Regime Flow in Pipeline
}

\author{
Guillermo Vilalta ${ }^{1}$, Mário Silva ${ }^{2}$ and Alejandro Blanco ${ }^{3}$ \\ ${ }^{1,2}$ Federal University of São João del Rei, Thermal Sciences and Fluids Department, 36307-352 São João del Rei - MG, Brazil. \\ ${ }^{3}$ Neurology and Neurosurgery Institute, Department of Engineering, Havana, Cuba.
}

\begin{abstract}
The drag reduction by polymer addition is wide interest in several areas. It has been shown that the polymer addition cushions the dissipative effects in turbulent flows. The main objective of this work is to establish a methodology for the numerical simulation of viscoelastic fluid through internal subroutines implemented in the Fluent code, via UDF. The validation of this methodology is made for the laminar flow regime case in pipeline. To describe the viscoelastic effect, it was used the Finitely Extensible Nonlinear Elastic model closing with Peterlin model. To taking in account the viscous effects $50 \leqslant \operatorname{Re} \leqslant 2000$ values were used. In addition, for the polymer concentration analysis it was used values which depend on the polymers molecular weight and the solution concentration that ranged from $0 \leqslant \mathrm{Cw} \leqslant 20$. The molecular elasticity and extensibility were maintained at constant values. The results showed that the addition of polymers regardless of their molecular weight in laminar flow regime causes no change in power dissipation. This result, which is consistent with the literature, is a significant advance in defining a credible and appropriate methodology to viscoelastic fluid flow study by UDF implementation of constituent models that characterize these fluids.
\end{abstract}

\section{Introduction}

An ideal elastic material, under pressure, responds with reversible deformation. When the tension ceases, the body returns to its original shape and volume. A viscous material when subjected to a shear stress, responds irreversibly to deformations, dissipating energy. The viscoelastic fluid undergoes both effects, dissipates some energy and stores the rest. These properties of viscoelastic fluids, has caused a significant increase of its practical applications, mainly those related with the fluid flow in internal flows, particularly in long distance transport fluid. It has been shown that the addition of a small amount of polymer, surfactant or other substances can cause relevant reductions on the resistance force, which leads to a considerable reduction of the power consumption in pumping systems. [1-3].

The first evidence of drag reduction due to certain additives, in particular polymers and/or fibers, was reported by Toms [1]. His results showed that the addition of $10 \mathrm{ppm}$ (by weight) of polymethyl methacrylate in a monochlorobenzene flow with high Reynolds number reduced substantially (30-40\%) the pressure drop compared to the pure solvent at the same flow rate. Other later studies confirmed this phenomenon, which has become known in the literature as Toms phenomenon. Despite the development of new techniques of visualization and mapping flows and measurement instruments, even today, there is no consensus on what is the real influence of the addition of polymers on the flow structure.

The drag reduction depends on the type of polymer and its concentration. Experimental and numerical studies have demonstrated that the drag reduction increases with increasing concentration of the polymeric solutions [2] and [3], but it can not exceed an upper limit, which is called the "Maximum Drag Reduction Asymptote" (MDRA). This curve is independent of the polymer concentration or its characteristic [4].

Furthermore, the drag reduction by polymer addition depends not only the concentration but also the kind of polymer, its molecular weight and the flow regime.

The drag reduction becomes increasingly effective at high molecular weight.

In addition, there is a consensus in the researches conducted to date that it is possible to check that the effect of drag reduction, by polymers injection in pipes, is highly effective in turbulent regime, but does not seem to have an effect on laminar flow regime [3], [5], [6].

Zhang et al [7] have demonstrated by numerical simulations that the polymer solutions flow using FENE$\mathrm{P}$ model in a sudden expansion geometry of $1: 3$ ratio and a rectangular cross section, in a laminar regime does not lead to a drag reduction. Oliveira [8] had also simulated for the same geometry and conditions of Zheng et al [7], but using DNS (Direct Numerical Simulation) and the FENE-CR viscoelastic model. His results from the point 
of view of the drag reduction coincide with the Zheng et al [7].

The major problem appears when the simulation of viscoelastic fluids because most commercial software does not have models for such fluids. This research has the aim of developing a methodology for simulation of viscoelastic fluids for consider a conformational model that allows modeling the polymer accurately and efficiently in confined flows. In order to do that it is intended to define an internal subroutine to be implemented in Fluent code, via UDF, in a way to incorporate the scalar equations and governing equations source terms for the calculations. For the first verification test of this methodology it was selected the simplest case that consist in a laminar flow in pipe of viscoelastic fluids at different concentrations defined by the FENE-P conformation model.

\section{Numerical simulation}

\subsection{Problem description}

It was simulated the flow inside a pipe with a commercial diameter of $2 "(50.8 \mathrm{~mm})$ and a unit of length. It is not necessary a greater length of pipe because it was applied the fully developed velocity profile at the entrance according to the Reynolds number, programmed by the UDF [9].

The mesh used in the simulations can be seen in Figure 1. It can be noted that near to the wall there is a further refinement of the grid, because this is a region of utmost importance in drag reduction question. More information at Silva [9].

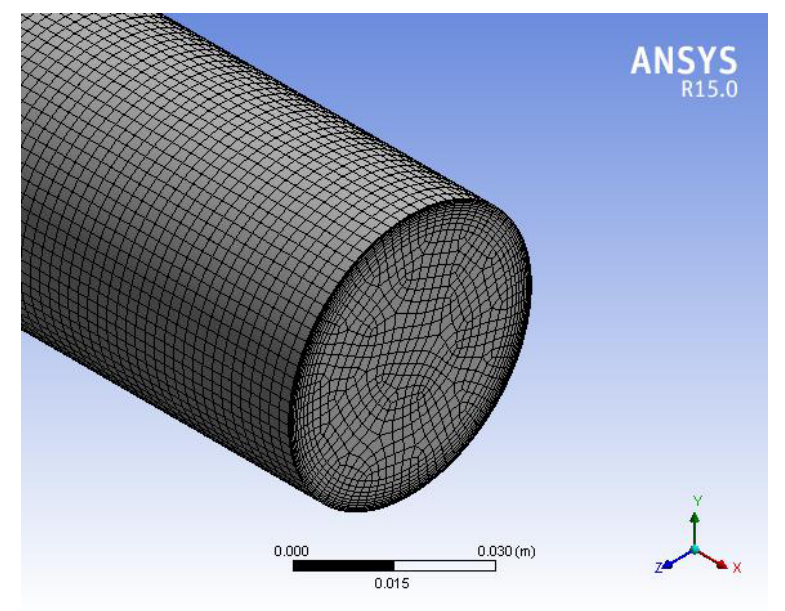

Figure 1. Mesh used in this study.

\subsection{Government equations}

When compared with the Newtonian fluids, it is remarkable the need for a more sophisticated constitutive relation to evaluate the stress tensor for viscoelastic fluids. FENE-P was the model used in this study because it is one of the polymers models used with some success in fluid dynamics simulation (CFD) since it eliminates the necessity of determining the statistical mean at each grid point in any moment in time. This model demonstrates to be able to capture some of the most important behaviors of polymeric flows such as drag reduction due to polymer addition in pipes [10]. In addition, FENE-P it is a nonlinear model, which represent more accurately the features of viscoelastic fluids.

For FENE-P model, the continuity equation is unchanged compared to a Newtonian flow:

$$
\frac{\partial \rho}{\partial t}+\frac{\partial}{\partial x_{i}}\left(\rho u_{i}\right)=0
$$

Where $u_{i}$ is the local instantaneous velocity component, $\rho$ is the fluid density, $x_{i}$ is the Cartesian coordinate component and $t$ is the time. In the other hand, the Navier-Stokes equations change, because of the stress caused by the polymer in the flow, emerging a new term related to the solute [7]:

$$
\rho \frac{\partial u_{i}}{\partial t}+\rho u_{j} \frac{\partial u_{i}}{\partial x_{j}}=-\frac{\partial p}{\partial x_{i}}+\frac{\partial \tau_{i j}{ }^{s}}{\partial x_{j}}+\frac{\partial \tau_{i j}^{p}}{\partial x_{j}}
$$

Where $p$ is the pressure, $\tau_{i j}{ }^{s}$ is the stress caused by the solvent being exactly the same Newtonian fluid flow stress (Equation (3)), and $\tau_{i j}{ }^{p}$ is the elastic stress caused by the polymer (Equation (4)):

$$
\begin{gathered}
\tau_{i j}{ }^{s}=\mu_{s}\left(\frac{\partial u_{i}}{\partial x_{j}}+\frac{\partial u_{j}}{\partial x_{i}}\right) \\
\tau_{i j}^{p}=N(x, t) \frac{\mu_{p}}{\lambda}\left[f(r) C_{i j}-\delta_{i j}\right]
\end{gathered}
$$

$C_{i j}$ is the tensor conformation component of the polymer molecule, $\mu_{s}$ is the dynamics solvent viscosity, $N(x, t)$ is the unit concentration and it is equal to 1 when the polymer or surfactant concentration is homogeneous along the flow (which is the case under consideration), $\mu_{p}$ is the solution viscosity, $\lambda$ is the polymer time relaxation, $\delta_{i j}$ is the Kronecker delta and $f(r)$ is the Peterlin function expressed by:

$$
f(r)=\frac{L^{2}-3}{L^{2}-r_{m o l}{ }^{2}}
$$

In the equation above, $L$ is the molecular (or micellar) extensibility polymer in relation to its equilibrium length. $r_{m o l}$ is the molecular (or micellar) length at steady state, and it is equal to the square root of the conformation tensor thread:

$$
r_{m o l}^{2}=\sum C_{i i}
$$

$C_{i j}$ can be formed from the inner product vector distance $\mathbf{r}$ from end to end molecule. Furthermore, according to Zhang et al [7] for this model $C_{i j}=C_{j i}$. In the "coiled" state (not extended or spiral) the polymers are usually spherical and $C_{i j}$ can be normalized: $C_{i j}{ }^{e q}=$ $\delta_{i j}$. The tensor conformation transport equation $C_{i j}$, also according to Zheng et al [7], is:

$$
\begin{aligned}
& \frac{\partial C_{i j}}{\partial t}+u_{k} \frac{\partial C_{i j}}{\partial x_{k}}=C_{i k} \frac{\partial u_{j}}{\partial x_{k}}+C_{k j} \frac{\partial u_{i}}{\partial x_{k}}-\frac{1}{\lambda}\left[f(r) \delta_{i k} C_{k j}-\right. \\
& \left.\delta_{i j}\right]+\Upsilon \frac{\partial}{\partial x_{k}}\left(\frac{\partial C_{i j}}{\partial x_{k}}\right)
\end{aligned}
$$


The last term of this transport equation is the artificial viscosity term (which is attached to diffusivity) which is added to increase the stability and convergence of the calculation. In the present study, a convergent solution can be obtained with $\Upsilon=0$ adjusting the sub-relaxation factors in the numerical simulations.

The Weissenberg number, We, expresses the relationship between the fluid relaxation time and a specific temporal process:

$$
W e=\dot{\gamma} \lambda=\lambda \frac{U}{D}
$$

Where $D$ is the pipe diameter, $U$ is the mean velocity and $\dot{\gamma}$ is the strain rate. For a polymer solution, the Reynolds number becomes [7]:

$$
R e=\frac{\rho U D}{\mu_{p}+\mu_{s}}
$$

Finally, another important parameter also in the viscoelastic model is the relationship between the viscosities of the pure solvent and polymer solution, as shown in Equation (10).

$$
\beta=\frac{\mu_{s}}{\mu_{s}+\mu_{p}}
$$

\subsection{Definition of parameters for simulations}

To evaluate the influence of the material type in the viscoelastic fluids flow in the simulations it was used two types of polymer, one with a high molecular weight (commercially called Iqapol) and another one with a low molecular weight (commercially called Iqapac) varying their concentrations in weight, $C_{w}$. As the solvent, it was used water, which is a highly fluid used in the industry.

According to Vilalta [3], the polymeric solution density formed by the polymers here used does not vary substantially. So it is necessary only the viscosity coefficient of the solutions determined by the polymer concentrations. Thus the viscosity values used herein are according to the values found by Vilalta [3] as a function of shear rate how is shown in Table 1. And for the density it was used the constant value $\rho=998,2 \mathrm{~kg} / \mathrm{m}^{3}$, which corresponds to the water density at ambient conditions of $20^{\circ} \mathrm{C}$ temperature and $1 \mathrm{~atm}(101.325 \mathrm{~Pa})$ pressure.

To take into account the viscous effects, the Reynolds number ranged between 50 and 2000 were used, so it can characterize the laminar regime and allowing understanding the viscoelastic fluid behavior near the transition zone.

The molecular extensibility was kept constant, $L^{2}=100$, which is a typical value for polymer solutions, since it allows considerable molecular extension before the fully extended state be achieved. Different values of 100 there is a very small influence on the flow [8].

The Weissenberg number was also kept constant and equal to 2, because according to Silva et al [9] by increasing the value of $W e$ it increases concomitantly the difficulties of convergence and the numerical method instability.
Thus, the values for the parameters discussed above were adopted according to Table 1.

Table 1. Parameters used in the studied.

\begin{tabular}{|cccc|}
\hline \multicolumn{4}{|c|}{ Iqapol (High molecular weight) } \\
\hline$C_{\boldsymbol{w}}(\mathbf{p p m})$ & $\boldsymbol{\mu}_{\boldsymbol{p}}(\boldsymbol{m P a} \boldsymbol{s})$ & $\boldsymbol{\beta}$ & $\boldsymbol{R} \boldsymbol{e}$ \\
\hline 5 & 1.309 & 0.434 & 50 \\
\hline 10 & 1.45 & 0.410 & 100 \\
\hline 15 & 1.645 & 0.378 & 500 \\
\hline 20 & 1.78 & 0.360 & 2000 \\
\hline \multicolumn{4}{|c}{ Iqapac (Low molecular weight) } \\
\hline $\boldsymbol{C}_{\boldsymbol{w}}(\mathbf{p p m})$ & $\boldsymbol{\mu}_{\boldsymbol{p}}(\boldsymbol{m P a} \boldsymbol{s})$ & $\boldsymbol{\beta}$ & $\boldsymbol{R} \boldsymbol{e}$ \\
\hline 15 & 1.21 & 0.453 & 50 \\
\hline 18 & 1.30 & 0.435 & 100 \\
\hline 20 & 1.37 & 0.423 & 500 \\
\hline \multicolumn{5}{c}{} & 2000 \\
\hline
\end{tabular}

The head loss $\left(h_{f}\right)$ in a flow of Newtonian fluid inside pipes can be calculated according to Equation (11) [11].

$$
h_{f}=\Delta z+\frac{\Delta p}{\rho g}+\frac{U_{1}{ }^{2}-U_{2}{ }^{2}}{2 g}=f \frac{L}{D} \frac{U^{2}}{2 g}
$$

Where $\Delta z$ is the topological difference between the inlet and outlet pipe, $\Delta p$ is the pressure difference also between the input and output, $U_{1}$ and $U_{2}$ are the mean velocities at the inlet and outlet pipe respectively, $L$ is the pipe length, $g$ is the local gravity acceleration and $f$ is the friction coefficient, which is a dimensionless parameter used to calculate the pressure drop in pipeline.

As the behavior of the flow is associate to the value of $f$, the variation of this parameter was selected to comparing the Newtonian and viscoelastic fluids to analyze the drag reduction. For the particular case of the laminar flow regime, the friction coefficient of a Newtonian fluid is analytically given by Equation (12) [11].

$$
f=\frac{64}{R e}
$$

\subsection{Numerical method and boundary conditions}

At the pipe inlet, a fully developed velocity profile was programmed and implemented, via UFD, as inlet boundary condition, according to each value of the Reynolds number analyzed. Using the Neumann boundary condition at the inlet and using the derivative value equal to zero, the velocity profile for the viscoelastic fluid flow by using FENE-P method is the same as the Newtonian fluid [9]:

$$
u=2 U\left[1-\left(\frac{r}{R}\right)^{2}\right]
$$

Where $R$ is the pipe radius and $r$ is the radial coordinate. At the outlet, it was selected the boundary condition linked to the pressure value, which assigns $0 \mathrm{~Pa}$ relative value. In the other words, it receives the absolute value of the reference pressure defined in the software such as atmospheric pressure with 101.325 Pa. Finally, in 
the wall region was defined the wall condition which imposes a non-slip condition.

The algorithm provided by Fluent software is based on the finite volume method. Double precision was used in the calculations. The convergence criteria (residuals) adopted for all variables was $10^{-5}$ and different underrelaxation factors were adopted for different conditions.

For spatial discretization of the momentum, continuity and transport equations for the tensor conformation was used the second order Upwind method. For the gradients numerical calculation, it was used the Least Squares Method Cell-Based Gradient.

For time discretization, it was used a second order implicit method known as "Backward Differentiation", described in Ferziger \& Peric [12].

The Pressure-Implicit Method with Splitting of Operators (PISO) that is more appropriate transient regime was adopted to solve the pressure-velocity coupling, and the standard scheme was used to the pressure equation discretization.

The tensor conformation starts considering its equilibrium state, when no force acts in the flow. And when in a state of non-equilibrium, for the tensor conformation for all surfaces (inlet, outlet and wall) it was adopted the Neumann boundary condition with derivatives equal to zero.

\section{Results}

\subsection{Polymer concentration effect}

Polymeric solutions concentration is certainly one of the most important parameters in respect to the drag reduction phenomenon by polymer addition. As abovementioned, the drag reduction tends to increase with concentration increasing, as was found in Lumley [2] and Vilalta [3] studies. However, both studies are related to the turbulent flow regime.

The analysis of the polymer concentration effects on friction coefficient and in head loss was carried out by mean of two representative cases. Case 1 represents a polymeric solution formed with a high molecular weight polymer, varying the polymer concentration according to Table 1 and keeping constant the Reynolds number $(R e=50)$, the elasticity parameter $(W e=2)$ and molecular extensibility $(L=10)$. Case 2 represents a polymeric solution formed with a low molecular weight polymer, varying the polymer concentration according to Table 1 and keeping constant the Reynolds number $(R e=500)$, the elasticity parameter $(W e=2)$ and molecular extensibility $(L=10)$. The Newtonian cases were simulated using the same Reynolds number for each case above defined.

Figure 2(a) shows the relation between the friction coefficient and the polymeric solutions concentration. It is possible to observe that the friction coefficient remains essentially unchanged with the concentration variation and no significant difference exist when compared with Newtonian fluid. This result is in agree with the literature. In Figure 2(b) is shown the relation between the head loss and the concentration of the polymeric solutions. When the concentration increases ( $\beta$ decreasing), an increasing in head loss is verified.

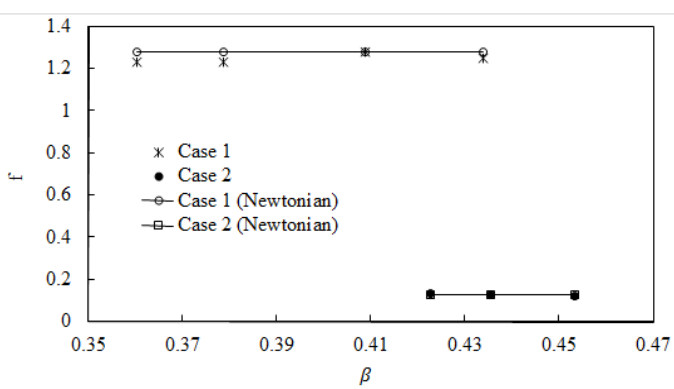

(a)

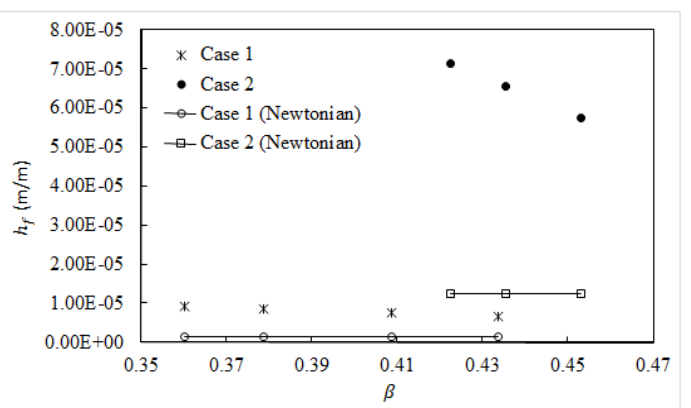

(b)

Figure 2. (a) Polymer concentration effect on the friction coefficient, $f$, for Cases 1 and 2; (b) Polymer concentration effect on the head loss, $h_{f}$, for Cases 1 and 2.

When compared with Newtonian fluid, the drag reducing effect is more significant in viscoelastic fluid. However, this is not directly associated to the effect of viscoelastic characteristics of the fluid. As already pointed out, no changes in friction coefficient in laminar flow of viscoelastic fluid.

The variation of head loss with the concentration of polymeric solution can be explained by the fact that the viscosity of viscoelastic fluid will always be higher than the Newtonian fluid, and tends to increase according to concentration increases. So, to keep constant the Reynolds number, the viscoelastic fluid flow velocity should increase proportionally with the viscosity. In the simulation here carried out, it is considered that the pipeline is horizontal $(\Delta z=0)$ and has transversal section constant. Therefore, the head loss is equals to pressure loss, as expressed by Equation (11). This means that the increase in velocity, with friction factor, diameter and length being constant, the head loss increases.

\subsection{Viscous effect}

For analysis of the viscosity effect, the Reynolds number was varied, taking into consideration that this dimensionless number is associated to the viscous forces acting in the flow. This analysis was carried out by means of Case 3 and Case 4.

Case 3 represents a polymeric solution formed with a high molecular weight polymer varying the $R e$ according to Table 1 and keeping the polymer concentration constant, $C_{w}=20$ ppm $(\beta=0,360)$. In the Case 4 , which corresponds to low molecular weight polymer, it 
was varied the $R e$ according to Table 1 and keeping the polymer concentration constant, $C_{w}=15 \mathrm{ppm}$ ( $\beta=$ $0,453)$. The Newtonian cases were simulated using the same $R e$ for each case defined above for pure water.

As might be expected, in all viscoelastic cases the curves follow the Newtonian case, so there is no drag reduction due to the polymer interference. These results are showed in Figure 3(a). It can also be observed from Figure 3(b) that the viscoelastic fluids have a loss of pressure greater than in Newtonian fluid in all cases examined. This is explained by the velocity increases that occurs in these fluids while keeping Reynolds number constant and not due to the friction factor $f$, as it was explained above in the analysis of the polymer concentration effect.

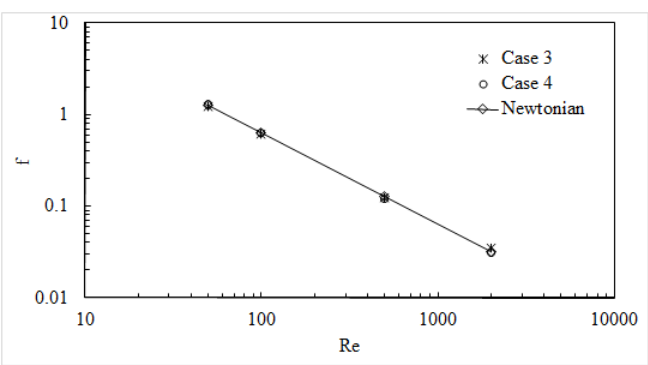

(a)

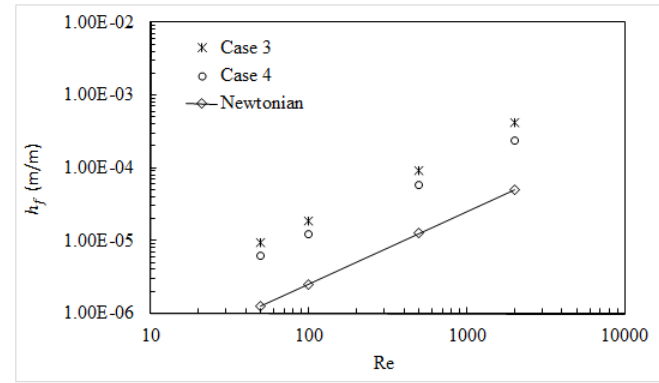

(b)

Figure 3. (a) Viscous effect in the friction coefficient, $f$, for Cases 3 and 4; (b) Viscous effect on the pressure loss, $h_{f}$, for Cases 3 and 4 .

These results are in agreement with those found experimentally by Virk (apud Diamond [5] and Wójs [6]), even in their case the latter study has modeled the viscoelastic fluid by the power law. No drag reduction effects were also verified by Zheng et al [7] and Oliveira [8] for a rectangular cross section in laminar regime.

\section{Conclusion}

This work represents a numerical investigation about the influence of the main fluid viscoelastic features on drag reduction phenomenon in laminar flow. The viscoelastic fluid was modeled by the FENE-P model.

The results obtained in this work show that in laminar flow the concentration and the kind of polymer do not interfere on drag reduction phenomenon. From drag reduction point of view, no changes in friction coefficient behavior were verified for all cases studied.

An interesting result was obtained when the influence of viscous effects on drag reduction was analyzed. When the pressure loss between Newtonian and viscoelastic fluid are compared, for the same values of Reynolds number, a higher loss pressure in viscoelastic fluids is observed. This result may seem contradictory because what is expected is a reduction in the pressure loss. As already indicated, to keep the Reynolds number constant, the mean velocity of the flow must increase. If no chang in the friction coefficient is verified, then an increase in the pressure loss should occur.

Establishing a numerical methodology, via UDF, as here presented, has important numerical challenges that are necessary solve, due to the inclusion of the conformation tensor transport equation. Such challenge concerns about the simulations convergence and numerical instabilities. All the challenges faced in this study were solved by using different strategies mainly related to appropriate selection of the time step and of the spatial discretization method. Different time steps were used for each of the cases studied. For numerical instabilities, second order Upwind model was used instead of one of third order. The latest one is more accurate than the second order Upwind, but has serious instability problems and is computationally more complex, especially when it comes to three-dimensional mesh.

The methodology here presented constitutes a significant advance in defining a credible and appropriate methodology for the study of viscoelastic fluid flow, by implementing the constitutive model. Further advances in this field will allow to extend the study to other flow regimes to get better insight of the inherent physic of the drag reduction phenomenon, aiming to extend their application in other industrial applications.

\section{References}

1. B. A. Toms, Anais Processing of The International Congress on Rheology, 135-141 (1949)

2. J. L. Lumley, Symp. Mathematica, 9, 315-334 (1972)

3. G. A. Vilalta, Ph.D. thesis, USP/Brazil (2000)

4. P. S. Virk, Aiche J. 21, 625-656 (1975)

5. P. Diamond, et al. Drag Reduction by Polymer Additives (The MITRE Corporation, Virginia, 1992)

6. K. Wójs, J. of Non-Newt. Fluid Mec. 48, 337-355 (1993)

7. Z. Zheng, F. Li, J. Yang, Hindawi Publishing Corporation, 2013, 1-13 (2013)

8. P. J. Oliveira, G. N. Rocha, R. J. Poole, J. of NonNewt. Fluid Mec. 114, 33-63 (2003)

9. M. L. F. Silva, Master dissertation, UFSJ/Brazil (2015)

10. A. P. Mósca, et al, IVth National Conference on Fluid Mechanics, Thermodynamics and Energy, 169178 (2012)

11. F. M. White, Mecânica dos Fluidos (Mc Graw Hill, Porto Alegre, 2011)

12. J. H. Ferziger, M. Peric, Computational methods for fluid dynamics (Rosewood, New York, 2002. 